\title{
Performance of binary exponential backoff CSMA in WiFi and optimal routing in mobile ad hoc networks
}

\author{
Philippe Jacquet, Amina Meraihi Naimi, Georgios Rodolakis \\ INRIA \& Ecole Polytechnique \\ France \\ philippe.jacquet@inria.fr, amina.naimi@inria.fr, georges.rodolakis@inria.fr
}

\begin{abstract}
In this paper we show that the CSMA IEEE 802.11 protocol (Wifi) provides packet access delays asymptotics in power law. This very feature allows us to specify optimal routing via polynomial algorithm while the general case is NP-hard.
\end{abstract}

Keywords: protocol performance, power law, delay routing, mobile ad hoc networks

\section{Introduction}

This paper addresses the problem of the evaluation of the packet delay delivery distribution via analytical means in a multihop wireless network. We assume that the wireless network operates with the IEEE 802.11 MAC protocol and implements the Optimal Link State Routing (OLSR) protocol [4]. The routing protocol is a table driven protocol that updates topology information via periodic broacast control packets. We denote by $W$ the end-to-end delay delivery of a packet.

A delay-oriented quality of service for a connection is generally expressed via two key parameters: a maximum acceptable delay $T$ and a maximum overdelay ratio $\epsilon$. The delay requirement states that during the connection one should have:

$$
P(W>T)<\epsilon
$$

Our aim is to present a protocol that evaluates the delay distribution $P(W>T)$ for any large $T$ (when $P(W>T)$ is small $)$ and uses it in order to find a route that satisfies the delay requirement of the connection.

In general finding the optimal route that minimizes an overdelay ratio is NP-hard [2]. Nevertheless we will show that the delay distribution at every node router is in power law and that this allows us to specify a polynomial approximation algorithm with error factor $1+O\left(T^{-1}\right)$.

\section{One hop delay estimate}

\subsection{Methodology overview}

Each wireless node can be seen as a buffer filled by incoming messages and served by a single server that performs the CSMA-CA multiple access protocol. We model this system as a M/G/1 system, i.e. we assume:

1. input packet flow in the buffer is Poisson of rate $\lambda$;

2. Service delays are independent

In fact the M/G/1 hypothesis is just a matter of simplifying approach. Since we are going to deal with heavy tailed distribution of service times, the consequence on queueing time distribution can be extended to a much larger class of queueing models. For example the power law in the tail delay distribution depends very little on the correlations between service times nor on the Poisson input assumption. What does matter in this case is the tail distribution of service and interarrival times. 


\subsection{Service delay determination}

We take the slotted time approach of [3]. IEEE 802.11 CSMA-CA protocol uses a rotating backoff where the nodes have to wait a random number of idle slots between transmission attempts. Let $\mathbf{C}$ be the random variable that expresses the number of busy slots between two consecutive idle slots. Let $p(L)$ the probability of collision that is experienced by packets whan they are of length $L$. The collision generally comes from hidden nodes and the longer is the packet the more likely it will experience such a collision. We take the following assumptions:

1. time between successive idle slots are independent and i.i.d;

2. collision events on successive transmissions are independent.

The CSMA protocol assumes that the backoff number is selected on an initial interval $\left\{1, \ldots, W_{\min }\right\}$. If a collision occurs the nodes in collision select a new backoff number on an enlarged interval $\left\{1, \ldots, 2 W_{\min }\right\}$, twice the length of the initial interval. Everytime a collision occurs for the retransmission of the same given packet, the node multiplies by two its backoff interval length. The backoff interval length is reset to $W_{\min }$ for any new packet to transmit. In practice there is a maximum number of retries after which the packet is discarded in case of permanent failure. The maximum retry number is 16 and leads to a delay that attains several seconds. Since this delay is larger than the practical acceptable delay for connection such as video or audio streams, this rule does not have practical impact on connection QoS, therefore it does not practically matter to set the maximum number of retries to infinity in the analytical model.

Let $C(z)$ be the probability generating function $\sum_{k} P(\mathbf{C}=k) z^{k}$, quantity $C$ being expressed in idle slot duration. Identity $C(z)=z$ would mean that $\mathbf{C}=1$ always, i.e the channel is permanently sensed idle. Let $\beta(z, L, p, k)$ be the probability generating function of the service delay when the packet length is $L$ and when the initial backoff interval is $k$. It satisfies the following recursion:

$$
\begin{aligned}
\beta(z, L, p, k)= & \frac{C(z)^{k+1}-C(z)}{C(z)-1} \frac{z^{L}}{k} \\
& \times(1-p+p \beta(z, L, p, 2 k)) .
\end{aligned}
$$

The delay probability generating function is $\beta(z)=E\left[\beta\left(z, L, p(L), W_{\min }\right)\right]$, averaging on packet length $L$ and collision probabilities $p(L)$. Figure 1 shows the 200 first coefficients of $\beta(z)$ when $C(z)=0.8 z+$ $0.2 z^{5}, L=4$ and $p=0.3$.

Theorem 1 We have, with $B=-\log _{2} p$ :

$$
\beta(z, L, p, k)=1+(1-z) v(1-z)+\left(k C^{\prime}(1) t\right)^{B} \alpha(\log (1-z))+O\left((1-z)^{B+1}\right)
$$

when $z \rightarrow 1$, where $v()$ is a polynomial and $\alpha()$ is a periodic function of period $\log 2$.

Proof: We fix $L$ and $p$ and set $e^{-\theta}=C(z)$ and denote $j(\theta, k)=\beta(z, k)$. We have

$$
j(\theta, k)=\frac{1-e^{-k \theta}}{k \theta} f(\theta)(1-p+p j(\theta, 2 k))
$$

with $f(\theta)=e^{\theta} \frac{\theta}{1-e^{-\theta}} z^{L}$. It is clear that $\theta=(1-z) C^{\prime}(1)+O\left((1-z)^{2}\right)$.

Defining $g(\theta)=\prod_{i \geq 1} \frac{1-e^{-\theta 2^{-i}}}{\theta 2^{-i}}$. Thus if $\nu(\theta, k)=g(k \theta) j(\theta, k)$, then

$$
\nu(\theta, k)=g(2 k \theta) f(\theta)(1-p)+p f(\theta) \nu(\theta, 2 k)
$$

And $\nu(\theta, k)=\left(\frac{1}{p}-1\right) \sum_{i \geq 1}(f(\theta) p)^{i} g\left(2^{i} k \theta\right)$.

It can be proven via classic application of Mellin transform over the logarithm, that function $g(\theta)$ is analytical and behaves like $1+O(\theta)$ when $\theta \rightarrow 0$ and converge to zero faster than any power law when $\theta \rightarrow \infty$.

Let $r_{B}(\theta)$ be the polynomial of degree $\lfloor B\rfloor$ which is the Taylor expansion of $g(\theta) e^{\theta}$ at $\theta=0$. Let $g_{B}(\theta)=g(\theta)-r_{B}(\theta) e^{-\theta}$. Clearly $g_{B}(\theta)=O\left(\theta^{\lceil B\rceil}\right)$ when $\theta \rightarrow 0$.

We have $\nu(\theta, k)=u_{B}(\theta)+\left(\frac{1}{p}-1\right) \sum_{i \geq 1}(f(\theta) p)^{i} g_{B}\left(2^{i} k \theta\right)$ with $u_{B}(\theta)=\left(\frac{1}{p}-1\right) \sum_{i \geq 1}(f(\theta) p)^{i} r_{B}\left(2^{i} k \theta\right) e^{-2^{i} k \theta}$. Clearly $u_{B}(\theta)$ is an analytical function with $u_{B}(\theta)=1+O(\theta)$.

Let $\nu_{B}(\theta, k)=\left(\frac{1}{p}-1\right) \sum_{i \geq 1}(f(\theta) p)^{i} g_{B}\left(2^{i} k \theta\right)$. We will show that $\mu_{B}(\theta, k)=\theta^{-B} \nu_{B}(\theta, k)$ is bounded when $\theta \rightarrow 0$. We have Let $h_{B}(\theta)=\theta^{-B} g_{B}(\theta)$. We have $\mu_{B}(\theta, k)=(1-p) \sum_{i \geq 1}(f(\theta))^{i} k^{B} h_{B}\left(2^{i} k \theta\right)$. 


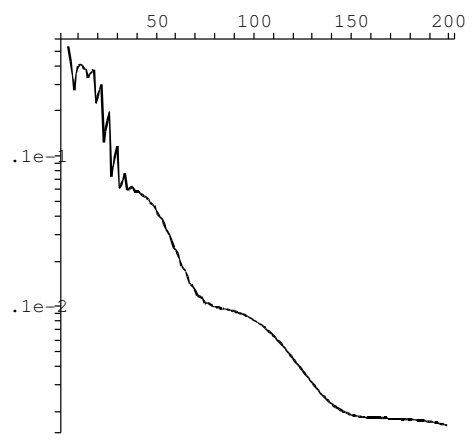

Fig. 1: Coefficients of $\beta(z)$

Since $f(\theta)=1+O(\theta)$, when $\theta \rightarrow 0$, we have $\mu_{B}(\theta, k)$ which converges to $\alpha(\log \theta)=k^{B} \sum_{i} h_{B}\left(2^{i} k \theta\right)$, the sum being on all integer $i$, including the negative integers. The sum converge because $h_{B}(\theta)=O\left(\theta^{\epsilon}\right)$ with $\epsilon=\lceil B\rceil-B$ and $h_{B}(\theta)$ decays faster than any power law. Notice that function $\alpha(x)$ is periodic of period $\log 2$.

Therefore $j(\theta, k)=\frac{\nu(\theta, k)}{g(k \theta)}$ has asymptotic expansion $\frac{u_{B}(\theta)}{g(k \theta)}+\alpha(\log \theta) \theta^{B}+O\left(\theta^{B+\epsilon}\right)$. Since $\frac{u_{B}(\theta)}{g(k \theta)}$ is analytical and equal to 1 at $\theta=0$, then it fits the required conditions.

\subsection{Delays including queueing}

We now investigate the overall packet delay including waiting delay in queue and service time. We assume that the buffer is infinite, the memory size is not a limiting factor in wireless nodes. We take the classic formula of probability generating function of waiting delay in a slotted M/G/1 queue $q(z)=$ $\sum_{k} P$ (packet waits $k$ slots $) z^{k}$ :

$$
q(z)=\exp \left((\beta(z)-1) \frac{\lambda}{2}\right) \frac{\left(1-\lambda \beta^{\prime}(1)\right)(1-z)}{1-z \exp (-(\beta(z)-1) \lambda)}
$$

This needs the provision that $\beta^{\prime}(1)$ exists. We will see that it implies that $p<\frac{1}{2}$. As well for the existence of the $k$ th moment of service time we need that $p<2^{-k}$. If $\lambda \ll 1$ then we could replace by

$$
q(z) \approx \frac{\left(1-\lambda \beta^{\prime}(1)\right)}{1-\frac{z}{1-z}(1-\beta(z)) \lambda}
$$

The generating function of the overall delay (queueing + service) of a packet of length $L$ with collision probability $p, w(z, L, p)$ satisfies the identity

$$
w(z, L, p)=q(z) \beta\left(z, L, p, W_{\min }\right) .
$$

Figure 2 shows the coefficients of $w(z)$ for $\lambda=0.02$. Notice that $\beta^{\prime}(1)=22.744 \cdots$.

\section{Asymptotic analysis of delay distribution}

Theorem 2 We have the expansion for $z$ around 1:

$$
\begin{aligned}
w(z)= & 1+(z-1) u(z-1) \\
& +\alpha(\log (1-z)) \frac{\left(W_{\min } C^{\prime}(1)\right)^{B} \lambda}{1-\lambda \beta^{\prime}(1)}(1-z)^{B-1} \\
& +O\left((1-z)^{B}\right),
\end{aligned}
$$

where $u(x)$ is an analytic function, $\epsilon>0$. 


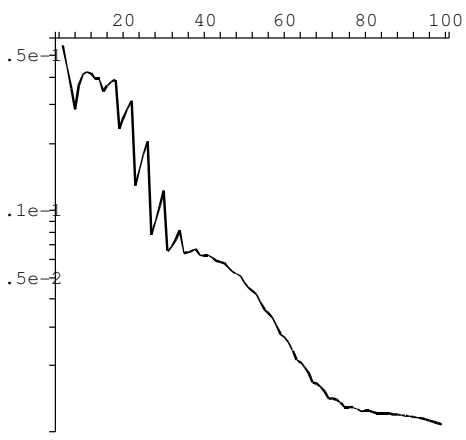

Fig. 2: Coefficients of $w(z)$

Proof: Easy derivation from previous theorem and expression (1).

Theorem 3 Applying Flajolet-Odlyzko tauberian theorems [1] on function $w(z)$, the probability that the delay in a router be greater than $T$, for $T$ large is

$$
\begin{aligned}
P(W>T)= & \alpha^{*}(\log T) \frac{\left(W_{\min } C^{\prime}(1)\right)^{B} \lambda}{1-\lambda \beta^{\prime}(1)} T^{1-B} \\
& +O\left(T^{-B}\right)
\end{aligned}
$$

where $\alpha^{*}(x)$ is a periodic function directly derived from function $\alpha(x)$ via its Fourier coefficients: if $\alpha(x)=\sum_{k} a_{k} \exp \left(\frac{2 i k \pi}{\log 2} x\right)$, then

$$
\alpha^{*}(x)=\sum_{k} \frac{a_{k}}{\Gamma(2-B-2 i k \pi / \log 2)} \exp \left(\frac{2 i k \pi}{\log 2} x\right) .
$$

Notice that the delay distribution tail decays in power law. As a corollary it turns out that the existence of the $k$ th moment of the delay needs the condition $p<2^{-k-1}$.

Proof: Application of Flajolet-Odlyzko Theorem [1].

Remark: The later assume non integer $B$, otherwise the conditions are more complex (involving singularities in $\log$ ).

\subsection{End-to-end delay}

We assume the following

- When traveling on its route, the delay experienced by a packet on a router is independent of the delay experienced on another router.

This assumption makes the problem easier to mathematically handle. But this may not realistic since the queues are necessarily correlated by two factors: (i) the flow packets which pass through the queues in tandem, (ii) the collision which results from common neighbor nodes are simultaneous. However it is not a fundamental assumption for our result since it is known that the sum of two random variables in power law, in particular the waiting times in queues, is still in power law whatever the dependence assumptions between them. The power law in the distribution function of the sum is simply the largest of the respective power laws of the terms, excepted that the factor in front of it will depend on the dependence assumptions. Assuming independence from now, if there are $n$ routers in the route from the source to the destination then the probability generating function of the end-to-end delay is equal to the product $\prod_{i \in \text { route }} w_{i}(z)$ where $w_{i}(z)$ is the probability generating function of the delay at router number $i$ and route is a set of router indices. Still with Flajolet Odlyzko result [1], if each $w_{i}(z)$ is of the form $1+$ $(z-1) u(z)+c_{i}(\log (1-z))(z-1)^{B_{i}}+O\left((z-1)^{B_{i}+1}\right.$, where $u_{i}()$ is analytic and $c_{i}()$ is of period 2 , then the leading term of $P(W$ (route $)>T)$ is $\sum_{i \in \text { route }} c_{i}^{*}(\log T) T^{1-B_{i}}$. Keeping only leading terms and ignoring small fluctuations of periodic functions for the sake of presentation: $P(W$ (route $)>T) \approx$ $c^{*}($ route $) T^{1-B(\text { route })}$, where $B($ route $)=\min B_{i}$ and $c^{*}($ route $)=\sum_{B_{j}=B} c_{j}^{*}$. 
An unexpected consequence of above is that a good choice for the route should not be the shortest path. In the shortest path the lap between two consecutive router maybe too large, leading to too large collision rates and therefore a too low value of $B$ (route). If one takes shorter hops between router then we will reduce the collision rate and get a larger value of $B$ (route). Of course this would be done in the detriment of a larger number of hops and a larger values of $c^{*}$ (route). But since in $c^{*}$ (route) $T^{1-B \text { (route) }}$, parameter

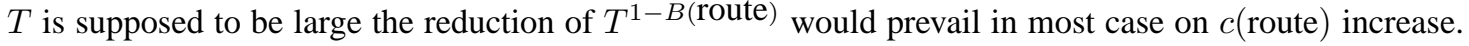
Interesting enough, increasing the number of hops and $c$ (route) will in most case increase the average end-to-end delay. Therefore we have the paradoxical case where increasing the average delay actually decrease the overdelay loss ratio. This is due to the fact that we expect the average delay to be much lower than the maximum acceptable delay $T$. Consequently routing with respect to average delay as it is done in [5] may conflict with the minimization of overdelay ratio.

Conversely the optimal route may be too long since it may have too short hops. In this case the connection may waste too many resource. Instead of choosing the route that minimizes $P(W>T)$ it is probably wiser to seek the shortest route that satisfies the requirement $P(W>T) \leq \epsilon$.

\section{Protocol implementation}

\subsection{Collision rates estimates}

OLSR uses hellos in order to detect neighbors. A node is neighbor if and only if the hello collison rate is below a given threshold $p_{0}$. Therefore OLSR has a procedure in advanced neighbor sensing option that allows to compute the collision rate (link quality level parameter). It uses the sequence number in order to identify the missing hellos. However there could be a difficulty in the fact that $p(L)$ may depend strongly on packet length $L$. One may expect a dependence of the kind $-\log p(L)=a L+b$ where $a$ and $b$ are scalar coefficients. Since the neighbor has no idea of the size of missing hellos, the transmitter should advertize the length distribution of its hellos. Comparing with its received hello distribution the neighbor would be able to determine the coefficient $a$ and $b$. By default the neighbor assume $b=0$, i.e. all packets have same collision rate regardless of its length.

\subsection{Advertizing Link quality}

it is preferable to use the option full-OLSR where the TC advertize the whole neighbor set instead of the MPR selector set. To this end a Link Quality Advertizement (LQA) message is used that is broadcasted via MPRs to the whole network. For each link $\ell$ it advertizes the collision rate $p_{\ell}$, and for the node itself it advertizes the global $\lambda$, the global collision rate and the local value of $C^{\prime}(1)$ (or only the tuple $\left(p, \frac{\lambda\left(W_{\min } C^{\prime}(1)\right)^{B}}{1-\lambda \beta^{\prime}(1)}\right)$.

\subsection{Delay based routing}

The problem is to find a route that satisfies the end-to-end delay requirement $P(W>T)<\epsilon$ of the new connection. We look to two directions. The first direction consists into finding the route that minimizes $P(W>T)$. The second direction consists into finding the shortest route that satisfies the requirement $P(W>T) \leq \epsilon$.

\subsubsection{Finding the optimal route}

In general finding the optimal route with respect to a delay distribution is NP hard [2]. But if we stick to asymptotic expression we can find a polynomial Dijkstra like algorithm. The idea is to find the route that provides the best asymptotic expansion of the quantity $P(W($ route $)>T)$ when $T \rightarrow \infty$. By best asymptotic expansion we mean the one provide asymptotically the lowest $P(W$ (route) $>T)$. Since we expect that $P(W$ (route) $>T)$ being asymptotically equivalent to $c^{*}$ (route) $T^{1-B \text { (route) }}$, the game consists into finding the route which minimizes the sum of the leading terms.

The Dijkstra algorithm is the following. The weight on the links is $c^{*}(\log T) T^{1-B}$. The weight of the route is the sum of the weights of the links:

$$
\sum_{i \in \text { route }} c_{i}^{*}(\log T) T^{1-B_{i}}
$$

The optimal route is the route that minimizes this sum. When $T \rightarrow \infty$ this would be the route that minimizes the tuple $\left(B\right.$ (route),$c^{*}$ (route) $)$ with the convention that $(B, c)<\left(B^{\prime}, c^{\prime}\right)$ iff either $B>B^{\prime}$ or $c<c^{\prime}$ when $B=B^{\prime}$. 


\subsubsection{Finding the shortest route}

As we seen before the shortest route that satisfies $P(W>T) \leq \epsilon$ is generally preferrable to the much longer route that minimizes $P(W>T)$. In the previous section we described a polynomial search of a which is optimal within a factor $1+O\left(T^{-1}\right)$. In fact for $T$ sufficiently large the search provides the optimal route. In the present section we want to find the shortest route according to a certain additive metric on link (the hop number) which satisfies a given constraint according to another additive metric (the quantities $c^{*}(\log T) T^{1-B}$ ). In general such multi-metric optimization problem is again NP-hard. However since the first metric can only take integer value we can easily make it polynomial. We describe the algorithm as follows. We consider a source node $A$.

Each node $B$ is assigned an integer vector:

$$
\left(p_{0}(A, B), p_{1}(A, B), \ldots, p_{n}(A, B)\right) .
$$

The value of $p_{i}$ is the smallest known value according to second metric of all routes of length $i$ according to first metric that connect node $A$ to node $B$. Clearly we have $p_{i}(A)=\min _{C \in \mathcal{N}(B)}\left(p_{i-1}(A, C)+\right.$ $p(C, B)$ )where $p(C, B)$ is the value of second metric on link $(C, B)$ and $\mathcal{N}(B)$ is the neighborhood of node $B$. Initializing all vectors to $(\infty, \infty, \ldots, \infty)$ excepted the vector of node $A$ itself set to $(0, \infty, \ldots, \infty)$, the algorithm will converge in $n$ steps (assuming that all comparisons in parallel in all nodes take one step). Notice that the coefficients $p_{i}(A, B)=\infty$ for all values of $i$ that are smaller than the distance between $A$ and $B$ (according to first metric). In order to get the route it suffices to manage a parallel vector that stores the optimal route of length $i$ for all $i$ from 0 to $n$.

\section{Conclusion}

We have analyzed Wifi which is the most popular wireless network protocol and we showed that the packet access control delay has a power law asymptotic distribution. This property is shown via generating functions and singularity analysis together with Flajolet-Odlyzko tauberian theorems. With respect to this property we have designed an adaptation of the popular routing protocol OLSR in order to achieve optimal routing according to over-delay constraint. In general overdelay constraint needs NP-hard algorithms, but in this case the asymptotics of the access delay allowed to design a polynomial approximation algorithm which is optimal when the delay threshold is large. We have found the paradoxical fact that over-delay constraints leads to significantly longer routes than with strictly shortest path algorithms. This comes from the fact that shorter hops leads to much smaller overdelay probabilities although the average delay increases. Therefore it is instrumental to have an algorithm that provides the only required over-delay and not the smallest over-delay probability, otherwise the path will require too many retransmissions and eventually affect the network capacity. Simulations show that the foundation asssumptions are robust enough that some be cleared of many simplifying hypothesis done so far throughout this paper, such as the independance between access delays between nodes and packets. Future works will be devoted in the design of more general protocol versions taking this into consideration.

\section{References}

[1] P. Flajolet, A. M. Odlyzko, "Singularity analysis of generating functions", SIAM J. Discrete Math. (1990) 3, 216-240

[2] R. Guerin and Ariel Orda. "QoS-based Routing in Networks with Inaccurate Information: Theory and algorithms." IEEE/ACM Transactions on Networking, Vol. 7, No. 3, June 1999, pp. 350-364.

[3] Bianchi "Perfomance Analysis of the IEEE802.11 Distributed coordination function" IEEE journal on selected areas in Communcations, vol 18, no 3, March 2000.

[4] T. Clausen, P. Jacquet, "the optimised link state routing (OLSR)," IETF RFC 3626.

[5] H. Badis, A. Munaretto, K. Al Agha, and G. Pujolle, "Optimal Path Selection in a Link State QoS Routing Protocol". IEEE VTC2004-spring, May 2004. 